\title{
5a-reductase activity in women with polycystic ovary syndrome: a systematic review and meta-analysis
}

\author{
Chuyan $\mathrm{Wu}^{1}$, Ke $\mathrm{Wei}^{2}$ and Zhongli Jiang ${ }^{1 *}$
}

\begin{abstract}
Background: 5a-reductase activity might be important during the development of polycystic ovary syndrome (PCOS). However, the changes of 5a-reductase activity in PCOS subjects and the relationship between 5a-reductase activity and body mass index (BMI), insulin resistance (IR) remain largely unknown.

Methods: We performed a meta-analysis to examine 5a-reductase activity in women with PCOS; exploratory subgroup analyses were also performed.

Results: Five articles (with 356 cases and 236 controls) reporting 5a-reductase activity in patients with PCOS were selected for the meta-analysis. We observed significantly higher ratios of 5aTHF/THF (5a-reduced tetrahydrocortisol to $5 \beta$-reduced tetrahydrocortisol) and An/Et (androsteroneto/etiocholanolone) levels, which were used to assess $5 \mathrm{a}$ reductase activity, among the patients with PCOS, [standardized mean differences (SMD) $=0.43$, 95\%confidence intervals $(95 \% \mathrm{Cl})=0.25-0.61, P<0.00001 ; \mathrm{SMD}=0.86,95 \% \mathrm{Cl}=0.29-1.44, P=0.003]$. We observed significant heterogeneity between studies for An/Et $\left(I^{2}=89 \%\right.$ and $\left.P<0.00001\right)$. According to the group analysis, women with PCOS exhibited increased 5 a-reductase activity which was significantly associated with homeostasis model assessment of insulin resistance (HOMA-IR) regardless of obesity.
\end{abstract}

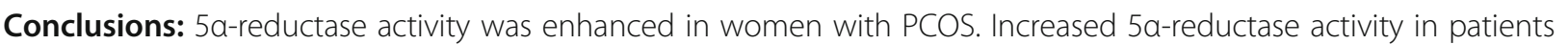
with PCOS was related to IR.

Keywords: 5a-Reductase, Polycystic ovary syndrome, Body mass index, Insulin resistance

\section{Background}

Polycystic ovary syndrome (PCOS) is a heterogeneous syndrome that occurs among women of reproductive age. PCOS affects $6-14 \%$ of women of reproductive age $[1,2]$. This syndrome is characterized by three main clinical features: hyperandrogenism, abnormal anovulation, and polycystic ovary morphology (via ultrasound) [3]. The diagnostic criteria for PCOS follows the National Institute of Health (NIH) criteria in 1992 [4] or the Rotterdam criteria in 2003 [5]. Hyperandrogenism is the critical factor of the pathophysiologic changes and clinical features associated with PCOS. In women with PCOS, excess androgen originates from the ovaries and

\footnotetext{
* Correspondence: jiangzh3721@163.com

${ }^{1}$ Department of Rehabilitation Medicine, the First Affiliated Hospital of

Nanjing Medical University, Nanjing, China

Full list of author information is available at the end of the article
}

adrenals [6]. An increased peripheral conversion of testosterone to the most active androgen $5 \alpha$-dihydrotestosterone ( $5 \alpha-\mathrm{DHT})$ occurs via $5 \alpha$-reductase regulation $[7,8]$. Longterm administration of androgens can induce visceral obesity and consecutive insulin resistance. Abdominal adiposity, insulin resistance and compensatory hyperinsulinemia could trigger androgenization in women [9].

$5 \alpha$-reductase is a key enzyme that is important to the metabolic processing of androgens and catalyzes the irreversible conversion of both cortisol to dihydrocortisol in the liver and testosterone to $5 \alpha$-DHT in the skin [10]. Two isoenzymes of $5 \alpha$-reductase have been described in previous studies. Increased type 1 isoenzyme is observed in skin and liver, and increased type 2 isoenzyme expression is mainly observed in reproductive tissues. The two types of isoenzymes are encoded by 5 alpha-reductase gene type 1 (SRD5A1) and 5 alpha- 
reductase gene type 2 (SRD5A2), respectively. It has confirmed that variants in SRD5A1 and SRD5A2 are associated with the prevalence of PCOS among lean women [11]. And increased $5 \alpha$-reductase activity is present in girls who are at risk for developing PCOS in early childhood [12]. Therefore, changes in 5a-reductase activity might be involved in the pathogenesis of PCOS [11], causing abnormal androgen levels, which is the critical factor of this syndrome.

Some studies have reported significantly increased $5 \alpha$ reductase levels among subjects with PCOS compared with controls [7, 13-16]. Overexpression of 5 $\alpha$-reductase is also observed in granulosa cells and cultured fibroblasts from patients with PCOS $[17,18]$. However, conclusions from similar studies differed. Some large population-based studies reported no differences in the metabolic components of $5 \alpha$-reductase in $24 \mathrm{~h}$ urine samples or in $5 \alpha$ dihydrotestosterone/testosterone ratios between the experimental and control groups [19, 20]. Therefore, the objective of this study was to systematically assess the changes in $5 \alpha$-reductase activity in subjects with PCOS by combining the results of previous studies. We also investigated the relationship between obesity, IR and $5 \alpha-$ reductase activity via a meta-analysis.

\section{Materials and methods}

This present meta-analysis was performed based on the PRISMA statement (Preferred Reporting Items for Systematic Reviews and Meta-Analyses). (Additional file 1: Table S1) [21] The present literature includes the literature search, selection criteria, quality score assessment, data extraction and statistical analysis. No registered protocol was required.

\section{Literature search strategy}

A database search was conducted in June 2016. The publications were mainly derived from electronic databases, such as PubMed, Embase, and the Cochrane Library. Only English language and human studies were searched. The following MeSHs terms and their combinations were used to search for included studies: " $5 \alpha$-reductase," "5alpha-reductase," "5alpha reductase," "steroid 5 alpha reductase," "polycystic ovary syndrome," "polycystic ovary," "PCOS". Only studies with human subjects were searched, and we also performed manual searches within all relevant publications. Two reviewers $(\mathrm{CW}$ and KW) searched the key words in the electronic databases independently. Disagreements between the two reviewers regarding data abstraction in the selection process were resolved through discussion.

\section{Inclusion and exclusion criteria}

Two investigators selected articles that assessed changes $5 \alpha$-reductase activity in women with PCOS. Studies were identified according to the following criteria:
1. Original data were presented.

2. The diagnosis criteria for PCOS were the Rotterdam criteria [5] or the NIH criteria [4].

3. Both an experimental and body mass index- (BMI) matched control group were included.

\section{Exclusion criteria}

1. Means and standard deviations (SDs) (and we could not obtain the original data from the authors) were not reported.

2. Studies that measured mRNA levels of $5 \alpha$-reductase or measured $5 \alpha$-reductase levels in tissue.

3. Inclusion of subjects receiving medical treatment and oral contraception pills within 3 months before entry into the study.

4. Inclusion of subjects with other diseases.

5. Letters, case reports, and other forms of content.

\section{Quality score assessment}

The Newcastle-Ottawa Scale was used to evaluate the quality of each included study [22] with some modifications. According to the results of the NOS assessment, the scores ranged between 0 and 7 . When the NOS score $<4$, studies were considered "low-quality," "Medium" quality studies scored $\leq 5$. A quality score $>5$ was defined as "high" quality.

\section{Data extraction}

The data were retrieved from each selected study by the two investigators (CW and $\mathrm{KW}$ ) independently. The general information from each study was systematically extracted using a structured data extraction form: first author's name, published year, country, matched factors, mean BMI, HOMA-IR, diagnostic criteria for PCOS, measurement method, mean ratio of $5 \alpha$-reduced tetrahydrocortisol to tetrahydrocortisol ( $5 \alpha \mathrm{THF} / \mathrm{THF})$, mean ratio of androsterone ( $5 \alpha$-reduced androgen metabolite) to etiocholanolone (5 $\beta$-reduced androgen metabolite) $(\mathrm{An} / \mathrm{Et})$, which were used to assess the $5 \alpha$-reductase activity [23], and their standard deviations (SDs). When the ratios were expressed as medians or geometric values, authors of the original research were contacted to obtain the necessary data. The data retrieved by the two investigators were expected to be the same, and any difference was resolved by consensus.

\section{Statistical analysis}

Heterogeneity in the studies eligible for the meta-analysis was considered significant when $\mathrm{P}_{\mathrm{Q}}<0.1$ according to a chi-squared $\mathrm{Q}$ test or $\mathrm{I}^{2}>50 \%$ according to I-squared statistics. We used a random-effects model according to the results of heterogeneity analysis. To understand the relationship between $5 \alpha$-reductase activity and the 
characteristics of PCOS, we further used corresponding subgroup analyses, especially when heterogeneity was obvious. When subgroup analyses were performed, we divided the subjects into a normal group $\left(<25 \mathrm{~kg} / \mathrm{m}^{2}\right)$ and an over-weight group $\left(\geq 25 \mathrm{~kg} / \mathrm{m}^{2}\right)$ according to BMI. The index of HOMA-IR was categorized according to the cutoff values for metabolic syndrome (MS): HOMA1-IR > 2.3 [24]. Studies with data of HOMA not reported or not available were categorized as the NR group.

When heterogeneity was significant, we used a sensitivity analysis to determine the stability of the results. We examined the influence of each included study on the results by excluding individual studies one at a time $[2,25]$. The meta-analysis was conducted using the statistical software RevMan 5.2.7 (Cochrane Collaboration,http://www.cc-ims.net/RevMan). Subgroup analyses were performed using the statistical software Stata 12.0 (StataCorp, College Station, TX, USA).

\section{Results}

\section{Literature selection}

Using the outlined search strategy, a total 167 potentially relevant citations were obtained for review. Of the 167 citations, 74 were duplicates, and 77 were not relevant. After removing duplicate and irrelevant articles, 16 studies were selected to be full-text assessed. Of the 16 studies, 11 articles that did not fulfill the selection criteria were excluded (Additional file 2). In two articles, subjects were grouped according to BMI values. One group was the normal group $\left(\mathrm{BMI}<25 \mathrm{~kg} / \mathrm{m}^{2}\right)$, and the other group was the over-weight group $\left(\mathrm{BMI} \geq 25 \mathrm{~kg} / \mathrm{m}^{2}\right)$ $[15,19]$. Therefore, these two publications were treated as four studies regarding the ratio of $5 \alpha \mathrm{THF} / \mathrm{THF}$. Only one publication was separated into two studies regarding the ratio of An/Et [15]. Finally, 5 articles $(n=7$ studies) were selected for $5 \alpha \mathrm{THF} / \mathrm{THF}[13-16,19]$, and 5 articles ( $n=6$ studies) were selected for An/Et. Figure 1 shows the flowchart of article selection.

\section{Characteristics of the studies}

The baseline information, matched factors, diagnostic criteria, and general data are clearly shown in the selected studies (Tables 1, 2 and Additional file 3: Table S2). The seven studies eligible for the ratio of $5 \alpha \mathrm{THF} /$ THF included 592 participants (356 PCOS patients and 236 controls). Six case-control studies selected for the ratio of An/Et included the same participants. In three studies, PCOS was diagnosed using the $\mathrm{NIH}$ criteria $[13,14,19]$, and the remaining two studies utilized the Rotterdam criteria [15, 16]. The PCOS and control groups were matched according to BMI and age. Four studies used urinary gas chromatography/mass spectrometry (GC/MS) analysis to measure cortisol and its metabolites [13-16], and one study used radioimmunoassay to measure cortisol and its metabolites [19]. However, the mean ratios of $5 \alpha \mathrm{THF} / \mathrm{THF}$ and An/Et were significantly different across these included studies. Four of the studies reported a higher ratio of $5 \alpha \mathrm{THF} /$ THF in the subjects with PCOS compared with controls. The other three studies reported no difference in
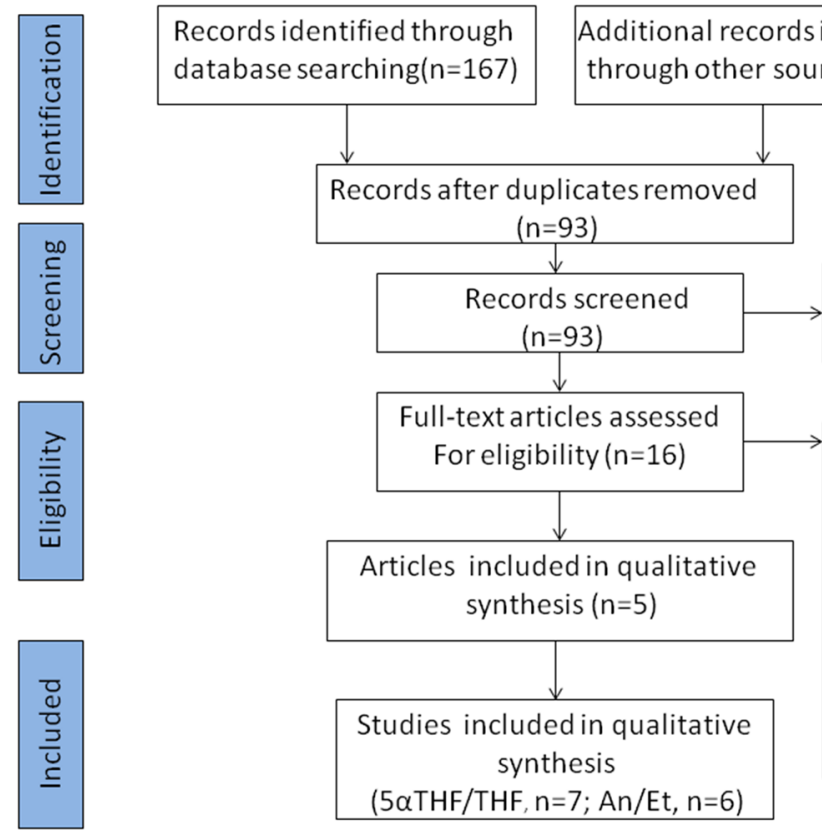

Records excluded according

To title and abstract: $(n=77)$

Full-text articles excluded With reasons:

1: BMI not matched

3: mRNA levers

4: Different methods for

the evaluation of

5alpha-reductase

3: Other diseases

(5 $\alpha$ THF/THF, $n=7 ; A n / E t, n=6)$

Fig. 1 Flow diagram of included studies 
Table 1 Characteristics of studies included in the meta-analysis for $5 a-$ THF/THF

\begin{tabular}{|c|c|c|c|c|c|c|c|c|c|c|}
\hline Study & Year & Country & Diagnosis Criteria & Npcos & Ncontrol & Matched factors & $\mathrm{BMI}$ & HOMA & $\begin{array}{l}5 a \text { reductase level } \\
\text { (5a-THF/THF) }\end{array}$ & NOS scores \\
\hline ANDREW RODIN & 1994 & UK & $\mathrm{NIH}$ & 34 & 18 & age, BMl & $\geq 25$ & $\mathrm{NR}$ & NS & 6 \\
\hline ANDREW RODIN & 1994 & UK & $\mathrm{NIH}$ & 31 & 27 & age, BMl & $<25$ & NR & NS & 6 \\
\hline D. Chin & 2000 & USA & $\mathrm{NIH}$ & 9 & 29 & age, BMl & $<25$ & $N R$ & $\uparrow$ & 5 \\
\hline TASOULA TSILCHOROZIDOU & 2003 & UK & $\mathrm{NIH}$ & 18 & 19 & age, BMI & $<25$ & 1.29 & $\uparrow$ & 6 \\
\hline Dimitra A. Vassiliadi & 2009 & UK & Rotterdam criteria & 75 & 28 & $\mathrm{BMI}$ & $\leq 25$ & 1.3 & $\uparrow$ & 5 \\
\hline Dimitra A. Vassiliadi & 2009 & UK & Rotterdam criteria & 103 & 72 & $\mathrm{BMI}$ & $>25$ & 3.1 & $\uparrow$ & 5 \\
\hline Michael W. O'Reilly & 2014 & UK & Rotterdam criteria & 86 & 43 & age, BMl & $>25$ & 2.3 & NS & 7 \\
\hline
\end{tabular}

Abbreviations: NIH National Institute of Health criteria, BMI body mass index, NR no reporting, NOS Newcastle-Ottawa Quality Assessment Scale

enzyme activity. Four of the studies observed higher An/Et ratios in women with PCOS. The other two studies did not observe a difference. In this meta-analysis, two publications ( $n=3$ studies) were defined as medium quality, and the quality scores were $5[13,15]$. The other three publications ( $n=4$ studies) $[14,16,19]$ were considered high quality.

\section{Meta-analysis}

The pooled analysis was performed with the 7 included studies that measured the ratio of $5 \alpha \mathrm{THF} / \mathrm{THF}$. When data from each study were included in the meta-analysis, the ratio of $5 \alpha \mathrm{THF} / \mathrm{THF}$ in women with PCOS was significantly higher than the BMI-matched controls (random-effects, $\mathrm{SMD}=0.43,95 \% \mathrm{CI}=0.25-0.61, P<0.00001$; Fig. 2). For the 6 included studies regarding the ratio of An/Et, the patients with PCOS exhibited significantly higher An/Et ratios than the BMI-matched controls (random-effects, SMD = $0.86,95 \% \mathrm{CI}=0.29-1.44, \quad P=0.003$; Fig. 3). However, significant heterogeneity was observed across the 6 studies eligible for the ratio of $\mathrm{An} / \mathrm{Et}\left(\mathrm{I}^{2}=89 \%\right.$ and $\left.P<0.00001\right)$.

Subgroup analysis: To understand the relationship between obesity, IR and the ratio of $5 \alpha \mathrm{THF} / \mathrm{THF}$ in patients with PCOS, subgroup analyses were performed. As shown in Fig. 4, the ratio of $5 \alpha \mathrm{THF} / \mathrm{THF}$ in the subjects with PCOS was significantly higher than in controls in all predefined categories for BMI, the diagnostic criteria of PCOS, and NOS scores. Significant differences were also observed both in HOMA-IR group and HOMA-NR group, but not in the group with the value of $\mathrm{HOMA}<2.3$.

In the subgroup analysis, significant differences in the ratio of $\mathrm{An} / \mathrm{Et}$ of the subjects PCOS versus controls were found in both the over-weight and normal groups $\quad(\mathrm{SMD}=1.00, \quad 95 \% \mathrm{CI}=0.04-1.96, \quad P=0.042$; $\mathrm{SMD}=0.72,95 \% \mathrm{CI}=0.01-1.43, \quad P=0.047$, respectively). There were significant differences in the ratio of An/Et in the quartiles with the diagnostic criteria of the $\mathrm{NIH}$ and Rotterdam criteria $(\mathrm{SMD}=1.40,95 \% \mathrm{CI}=0.26-$ 2.53, $P=0.016$; $\mathrm{SMD}=0.44,95 \% \mathrm{CI}=0.23-0.64, P=0.000$ ), also in the quartiles with HOMA-IR and HOMA-NR groups $\quad(\mathrm{SMD}=0.46, \quad 95 \% \quad \mathrm{CI}=0.23-0.70, \quad P=0.517$; $\mathrm{SMD}=2.02,95 \% \mathrm{CI}=1.59-2.45, P=0.306)$.

The subgroup analysis based on NOS scores revealed that significantly higher An/Et ratios were associated with medium-quality studies $(\mathrm{SMD}=0.97,95 \% \mathrm{CI}=-0.24-2.17$, $P=0.009 ; \mathrm{I} 2=72.9 \%, P=0.025$ for heterogeneity). However, there was no significant difference in the high-quality score studies $(\mathrm{SMD}=0.71,95 \% \mathrm{CI}=0.17-1.24, P=0.115$; $\mathrm{I}^{2}=92 \%, P<0.001$ for heterogeneity; Fig. 5).

\section{Sensitivity analysis}

When studies were omitted one by one, the total SMDs and $95 \%$ CIs for both the ratio of $5 \alpha \mathrm{THF} / \mathrm{THF}$ and $\mathrm{An} /$

Table 2 Characteristics of studies included in the meta-analysis for An/Et

\begin{tabular}{|c|c|c|c|c|c|c|c|c|c|c|}
\hline$\overline{\text { Study }}$ & Year & Country & Diagnosis Criteria & Npcos & Ncontrol & Matched factors & $\mathrm{BMI}$ & HOMA & $\begin{array}{l}\text { 5a- reductase level } \\
\text { (An/Et ratio) }\end{array}$ & NOS scores \\
\hline ANDREW RODIN & 1994 & UK & $\mathrm{NIH}$ & 65 & 45 & age, BMI & $\geq 25$ & NR & NS & 6 \\
\hline D. Chin & 2000 & USA & $\mathrm{NIH}$ & 9 & 29 & age, BMI & $<25$ & NR & $\uparrow$ & 5 \\
\hline TASOULA TSILCHOROZIDOU & 2003 & UK & $\mathrm{NIH}$ & 18 & 19 & age, BMl & $<25$ & 1.29 & NS & 6 \\
\hline Dimitra A. Vassiliadi & 2009 & UK & Rotterdam criteria & 75 & 28 & $\mathrm{BMI}$ & $<25$ & 1.3 & $\uparrow$ & 5 \\
\hline Dimitra A. Vassiliadi & 2009 & UK & Rotterdam criteria & 103 & 72 & $\mathrm{BMl}$ & $\geq 25$ & 3.1 & $\uparrow$ & 5 \\
\hline Michael W. O'Reilly & 2014 & UK & Rotterdam criteria & 86 & 43 & age, BMI & $\geq 25$ & 2.3 & $\uparrow$ & 7 \\
\hline
\end{tabular}




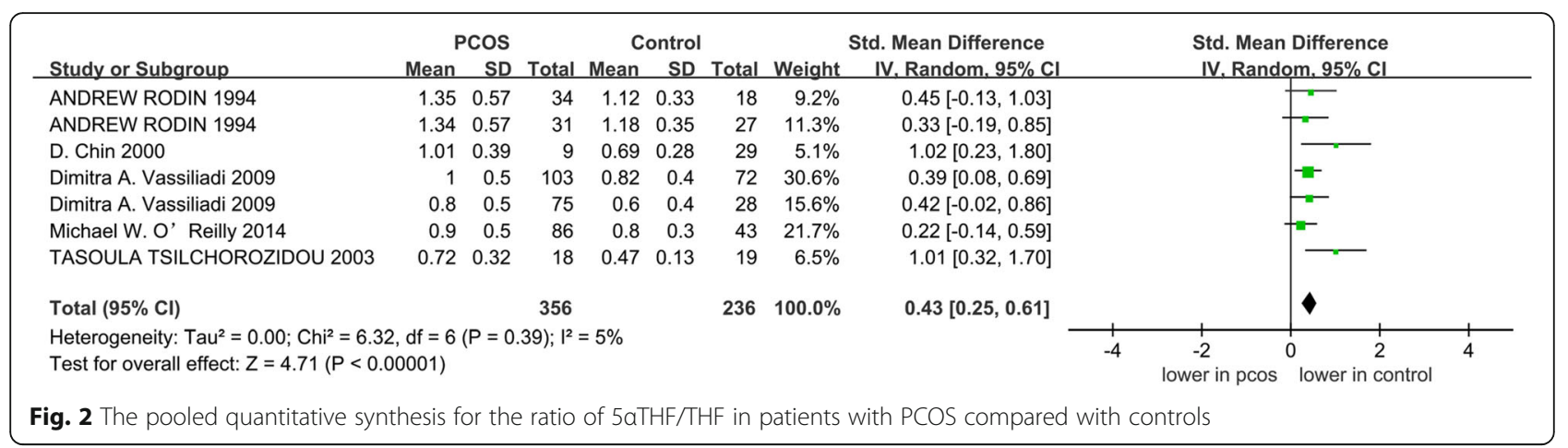

Et were not significantly changed. The study by Andrew Rodin et al. [19] utilized a different method measure cortisol and its metabolites. The authors reported impressive negative results whether for $5 \alpha \mathrm{THF} / \mathrm{THF}$ or An/Et. When this study was excluded, there were no obvious changes in the results $(\mathrm{SMD}=0.48,95 \% \mathrm{CI}=0.22-0.73, P<0.001$; $\mathrm{SMD}=0.53,95 \% \mathrm{CI}=0.23-0.82, \quad P<0.001)$. However, when the study was excluded, the heterogeneity across the 6 studies for the ratio of An/Et decreased significantly $\left(\mathrm{I}^{2}=49 \%\right.$ and $P=0.10$ for heterogeneity).

\section{Publication bias}

Because the number of studies included in this metaanalysis was fewer than 10 , we could not use a funnel plot or any other statistical analysis methods to assess the potential publication bias $[25,26]$.

\section{Discussion}

Although most of the studies had small sample sizes, the activity of $5 \alpha \mathrm{THF} / \mathrm{THF}$ and An/Et were significantly higher in subjects with PCOS when compared to BMImatched controls, and the results from group analysis suggested that high $5 \alpha \mathrm{THF} / \mathrm{THF}$ and An/Et levels were significantly associated with HOMA regardless of the degree of obesity or diagnostic criteria. The An/Et ratio between the subjects with PCOS and BMI-matched controls was not significantly different in the "high" quality studies, and there was significant heterogeneity across the 6 studies eligible for the ratio of An/Et.

Recently, pharmacological actions targeting cortisol metabolism as a therapeutic tool have attracted widespread attention. In PCOS women, increased $5 \alpha$ reductase activity has been associated with idiopathic hirsutism, androgenic alopecia, and acne. Increased $5 \alpha-$ reductase Activity would enhance cortisol metabolism resulting in a compensatory increase in $\mathrm{ACTH}$ secretion and stimulation of adrenal steroid-genesis. In women with PCOS, increased 5reductase activity in specific tissues, such as the skin and ovary, has been reported [12]. Even the daughters of women with PCOS have increased $5 \alpha \mathrm{THF} / \mathrm{THF}$ ratios [12], suggesting increased global $5 \alpha-$ reductase activity. In the overall analysis, we confirmed the enhanced $5 \alpha$-reductase activity in women with PCOS. Most patients with PCOS exhibit significant insulin resistance [27] and have changed $5 \alpha$-reductase activity $[7,28] .5 \alpha$-reductase is thought to play an important role in the formation of insulin resistance, which is a major clinical feature of PCOS. The findings of the previous study indicate that enhanced $5 \alpha$-reductase activity in both men and women is related to insulin resistance [28]. The results of our subgroup analysis also showed that the high levels of $5 \alpha$-reductase activity in IR groups. In addition, we studied the relationship between increased $5 \alpha$-reductase activity and obesity. Elevated $5 \alpha$ -

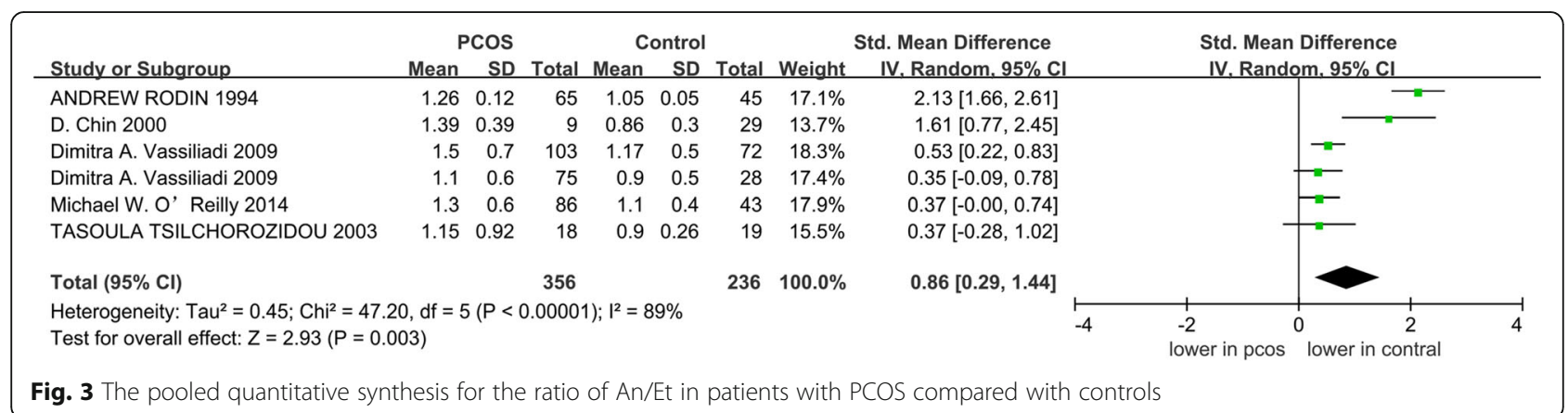




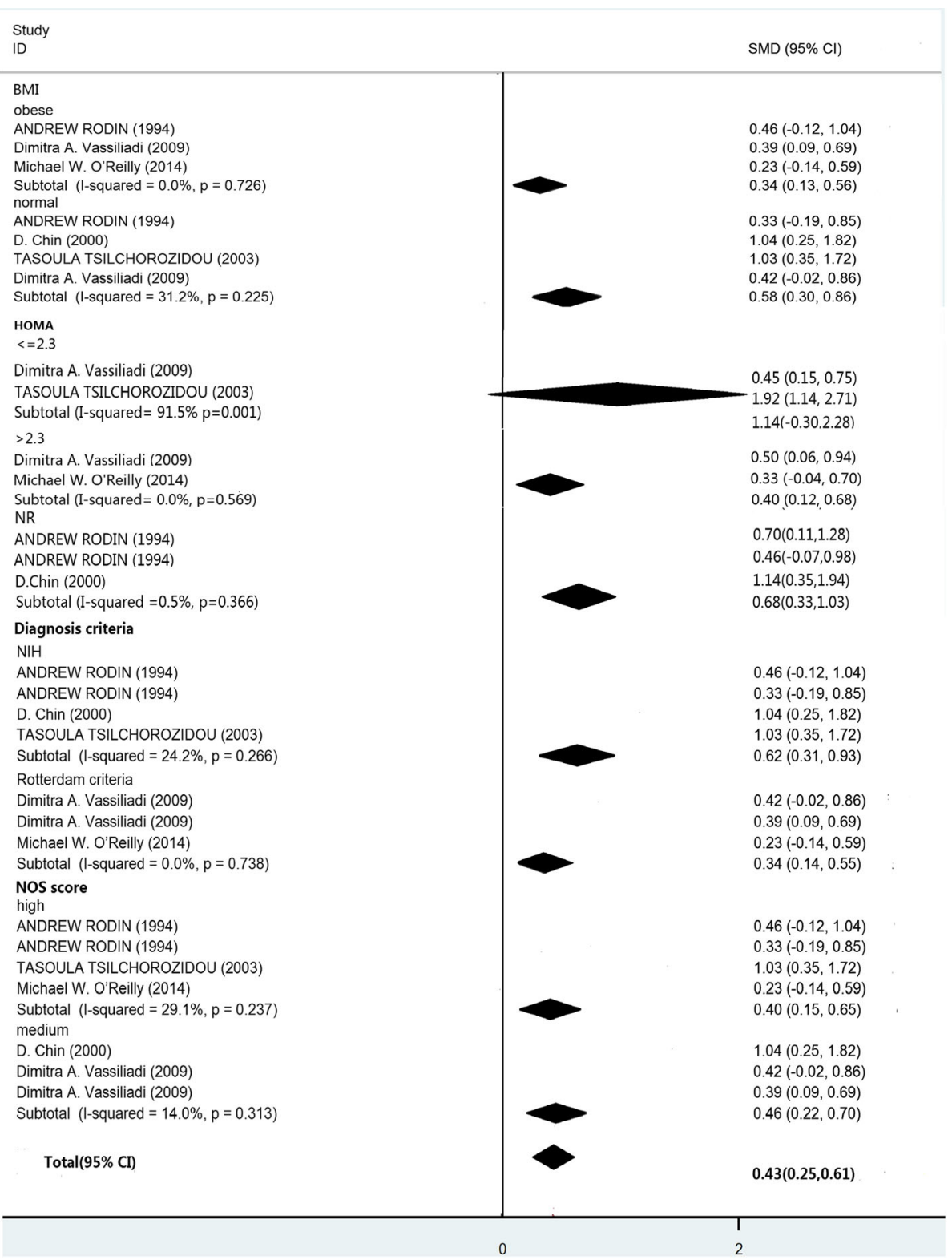

Fig. 4 Summary of categorical meta-analysis for 5aTHF/THF

reductase activity was observed in both the normal and over-weight groups of women with PCOS, suggesting that enhanced ratios of $5 \alpha \mathrm{THF} / \mathrm{THF}$ and An/Et are not associated with obesity. In some previous studies, $5 \alpha$ reductase was positively correlated with body weight in adult women with PCOS [14, 15]. But one study in 2011 reported genetic alterations in the $5 \alpha$-reductase gene in lean patients with PCOS, which lead to characteristic clinical changes [11]. Combined with the results of these previous studies, our results from the subgroup analysis suggest that obesity does not affect $5 \alpha$-reductase activity in patients with PCOS. Because of an extended span of time, our study did not have enough data to perform subgroup analyses of androgen. Further research is required.

It is the first meta-analysis regarding $5 \alpha$-reductase. Although there were some limitations, our study evaluated the association of the main characteristics of women with PCOS, such as obesity, IR and $5 \alpha$-reductase activity. Our meta-analysis could provide a direction for future research in this area. In addition, we strictly controlled the inclusion criteria of the articles in this metaanalysis, and we conducted rigorous quality scoring of the literature selected in the study according to NOS 


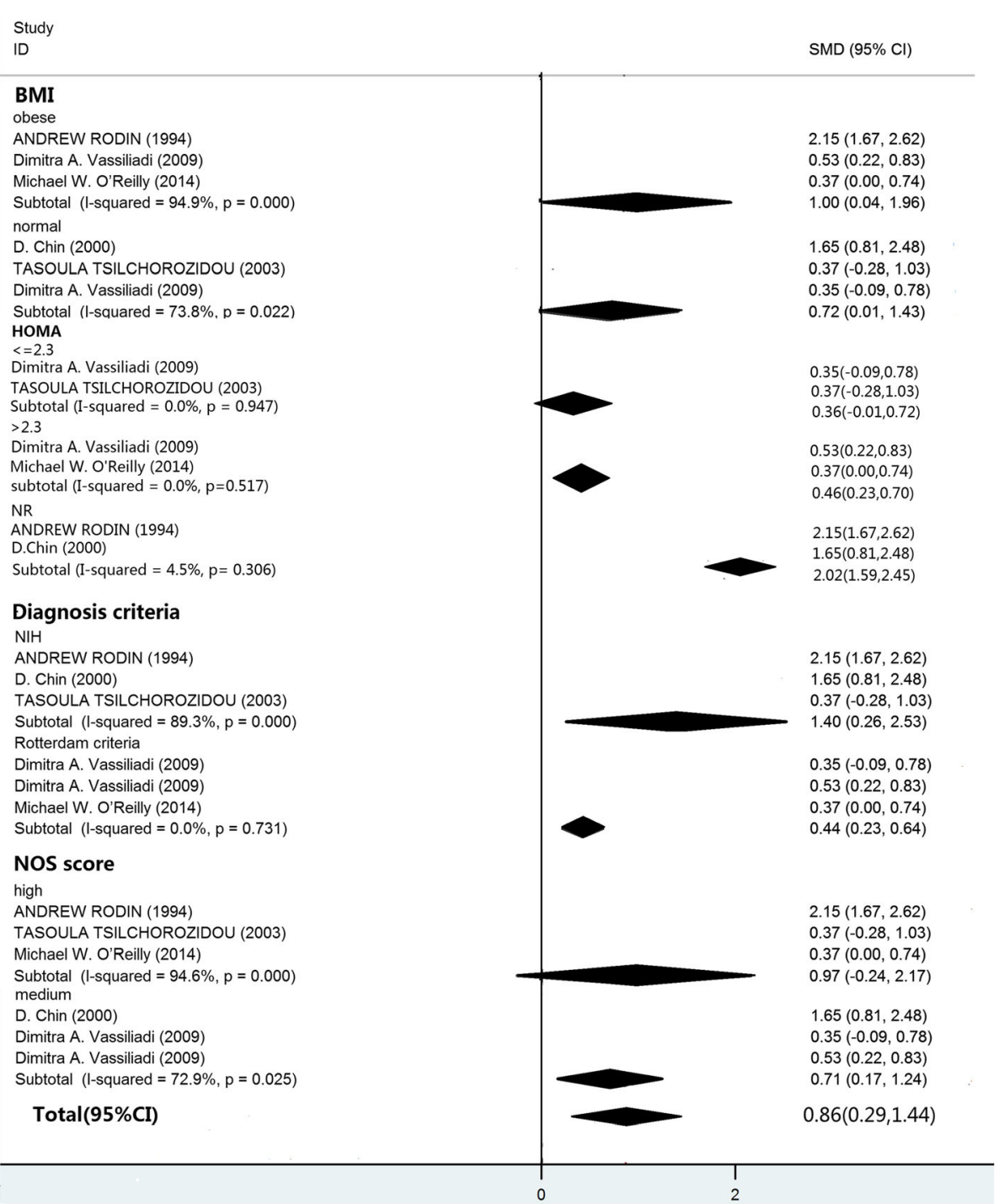

Fig. 5 Summary of categorical meta-analysis for An/Et

criteria. We used various methods to identify potential sources of heterogeneity. During the evaluation of diagnostic criteria for the subgroup analysis, we identified one heterogeneous study. However, the results were not affected after exclusion of this study. Therefore, our results are relatively stable.

There are some limitations that should be considered in this meta-analysis. First, significant heterogeneity was observed in the studies eligible for the ratio of $\mathrm{An} / \mathrm{Et}$, and the reliability of our findings might be reduced because of this heterogeneity. The included studies for the ratio of An/Et did not have detailed subgroups (because the heterogeneity was lost in the diagnostic criteria of Rotterdam when the subgroup analysis was performed). PCOS is a kind of endocrine disease with strong clinical heterogeneity. Moreover, the sample sizes in most of the selected studies were not large enough. Because of these influencing factors, it is easy to understand the emergence of significant heterogeneity in our meta-analysis for the An/ Et ratio. When we performed the sensitivity analysis, the heterogeneity was significantly decreased after one study was excluded, but the results remained unchanged. We analyzed this study, and the detection methods used for An and Et differed from the other studies, which might be the source for the existence of heterogeneity. However, this study did not cause significant heterogeneity regarding the $5 \alpha \mathrm{THF} / \mathrm{THF}$ ratios. Therefore, we did not exclude the study. Second, unpublished data cannot be included, and studies with negative results are difficult to publish. All these factors could lead to the emergence of publication bias in the analysis. 


\section{Conclusions}

In this meta-analysis, $5 \alpha$-reductase activity was enhanced in subjects with PCOS compared with BMI-matched controls, and enhanced $5 \alpha$-reductase activity in subjects with PCOS was associated with IR rather than obesity. Therefore, increased $5 \alpha$-reductase activity could be an important biochemical characteristic of PCOS. Given the limited number of studies included in the analysis, the findings from our meta-analysis should be confirmed in future research.

\section{Additional files}

Additional file 1: Table S1. PRISMA 2009 Checklist. (DOC 70 kb)

Additional file 2: The search strategy and excluded studies with reasons. (DOCX $28 \mathrm{~kb}$ )

Additional file 3: Table S2. Data from the studies included in the meta-analysis. (DOCX 17 kb)

\section{Abbreviations}

5a-DHT: 5a-dihydrotestosterone; 5aTHF/THF: 5a-reduced tetrahydrocortisol to $5 \beta$-reduced tetrahydrocortisol; $95 \% \mathrm{Cl}$ : $95 \%$ confidence intervals; $\mathrm{An} /$ Et: Androsteroneto/etiocholanolone; BMl: Body mass index; GC/MS: Gas chromatography/mass spectrometry; HOMA-IR: Homeostasis model assessment of insulin resistance; IR: Insulin resistance; MS: Metabolic syndrome; NIH: National Institute of Health; PCOS: Polycystic ovary syndrome; PRISMA: Preferred Reporting Items for Systematic Reviews and MetaAnalyses; SDs: Standard deviations; SMD: Standardized mean differences; SRD5A1: 5 alpha-reductase gene type 1; SRD5A2: 5 alpha-reductase gene type 2

\section{Acknowledgments}

None.

\section{Funding}

This study was supported by the National Nature Science Foundation of China (81501947). The support from the Priority Academic Program Development (PAPD) of Jiangsu Higher Education Institutions was also appreciated. The funders had no role in the study design, data collection and analysis, decision to publish, or preparation of the manuscript.

\section{Availability of data and materials}

Not applicable.

\section{Authors' contributions}

Conceived and designed the experiments: ZLJ. Performed the experiments: CYW and KW. Analyzed the data: CYW. Contributed reagents/materials/ analysis tools: ZLJ. Wrote the paper: CYW. All authors read and approved the final manuscript.

\section{Competing interests}

The authors declare that they have no competing interests.

\section{Consent for publication}

Not applicable.

Ethics approval and consent to participate

Not applicable.

\section{Publisher's Note}

Springer Nature remains neutral with regard to jurisdictional claims in published maps and institutional affiliations.

\section{Author details}

${ }^{1}$ Department of Rehabilitation Medicine, the First Affiliated Hospital of Nanjing Medical University, Nanjing, China. ${ }^{2}$ Medical Service Section, the First Affiliated Hospital of Nanjing Medical University, Nanjing, China.

Received: 25 November 2016 Accepted: 20 March 2017

Published online: 27 March 2017

\section{References}

1. Azziz R, et al. The prevalence and features of the polycystic ovary syndrome in an unselected population. J Clin Endocrinol Metab. 2004;89(6):2745-9.

2. Meng $Y$, et al. Association between High Serum Homocysteine Levels and Biochemical Characteristics in Women with Polycystic Ovarian Syndrome: a Systematic Review and Meta-Analysis. Plos One. 2016;11(6):e0157389.

3. Peng Z, et al. Interleukin-6 Levels in Women with Polycystic Ovary Syndrome: A Systematic Review and Meta-Analysis. Plos One. 2016;11(2):e0148531.

4. Zawadzki J, Dunaif A. Diagnostic criteria for polycystic ovary syndrome: towards a rational approach. Polycistic Ovary Syndr. 1992;4:377-84.

5. Rotterdam ESHRE/ASRM-Sponsored PCOS Consensus Workshop Group. Revised 2003 consensus on diagnostic criteria and long-term healthiest related to polycystic ovary syndrome. Fertil Steril. 2004;81(1):19-25.

6. Loughlin T, et al. Adrenal abnormalities in polycystic ovary syndrome. J Clin Endocrinol Metab. 1986;62(1):142-7.

7. Stewart PM, et al. 5 alpha-reductase activity in polycystic ovary syndrome. Lancet. 1990;335(8687):431-3.

8. Fassnacht $M$, et al. Beyond adrenal and ovarian androgen generation: Increased peripheral 5 alpha-reductase activity in women with polycystic ovary syndrome. J Clin Endocrinol Metab. 2003;88(6):2760-6.

9. Wu C, et al. The characterization of obese polycystic ovary syndrome rat model suitable for exercise intervention. Plos One. 2014;9(6):e99155.

10. Remer T, Maser-Gluth C, Wudy SA. Glucocorticoid measurements in health and disease-metabolic implications and the potential of 24-h urine analyses. Mini Rev Med Chem. 2008:8(2):153-70.

11. Graupp $M$, et al. Association of genetic variants in the two isoforms of 5alpha-reductase, SRD5A1 and SRD5A2, in lean patients with polycystic ovary syndrome. Eur J Obstet Gynecol Reprod Biol. 2011;157(2):175-9.

12. Torchen LC, et al. Evidence for Increased 5alpha-Reductase Activity During Early Childhood in Daughters of Women With Polycystic Ovary Syndrome. J Clin Endocrinol Metab. 2016;101(5):2069-75.

13. Chin D, et al. Increased 5alpha-reductase and normal 11 beta-hydroxysteroid dehydrogenase metabolism of C19 and C21 steroids in a young population with polycystic ovarian syndrome. J Pediatr Endocrinol Metab. 2000;13(3):253-9.

14. Tsilchorozidou T, Honour JW, Conway GS. Altered cortisol metabolism in polycystic ovary syndrome: insulin enhances 5alpha-reduction but not the elevated adrenal steroid production rates. J Clin Endocrinol Metab. 2003; 88(12):5907-13.

15. Vassiliadi DA, et al. Increased 5 alpha-reductase activity and adrenocortical drive in women with polycystic ovary syndrome. J Clin Endocrinol Metab. 2009;94(9):3558-66.

16. O'Reilly MW, et al. Hyperandrogenemia predicts metabolic phenotype in polycystic ovary syndrome: the utility of serum androstenedione. J Clin Endocrinol Metab. 2014:99(3):1027-36.

17. Magoffin DA. Ovarian enzyme activities in women with polycystic ovary syndrome. Fertil Steril. 2006;86 Suppl 1:S9-S11.

18. Boda D, Paun D, Diaconeasa A. Evaluation of 5-alpha reductase activity on cultured fibroblast in patients with hyperandrogenemia. Rom J Intern Med. 2009:47(1):67-73.

19. Rodin A, et al. Hyperandrogenism in polycystic ovary syndrome. Evidence of dysregulation of 11 beta-hydroxysteroid dehydrogenase. N Engl J Med. 1994;330(7):460-5.

20. Gambineri A, et al. Increased clearance of cortisol by 5beta-reductase in a subgroup of women with adrenal hyperandrogenism in polycystic ovary syndrome. J Endocrinol Invest. 2009;32(3):210-8.

21. Moher D, et al. Preferred reporting items for systematic reviews and metaanalyses: the PRISMA statement. BMJ. 2009;339:b2535.

22. Wells G, et al. The Newcastle-Ottawa Scale (NOS) for assessing the quality of nonrandomised studies in meta-analyses. Ontario: Department of Epidemiology and Community Medicine, University of Ottawa; 2000.

23. Tomlinson JW, et al. Reduced glucocorticoid production rate, decreased 5 alpha-reductase activity, and adipose tissue insulin sensitization after weight loss. Diabetes. 2008;57(6):1536-43. 
24. Geloneze B, et al. HOMA1-IR and HOMA2-IR indexes in identifying insulin resistance and metabolic syndrome: Brazilian Metabolic Syndrome Study (BRAMS). Arq Bras Endocrinol Metabol. 2009;53(2):281-7.

25. Egger $\mathrm{M}$, et al. Bias in meta-analysis detected by a simple, graphical test. BMJ. 1997;315(7109):629-34.

26. Bazarganipour F, et al. The impact of polycystic ovary syndrome on the health-related quality of life: a systematic review and meta-analysis. Iran J Reprod Med. 2015;13(2):61-70.

27. Legro RS, et al. Prevalence and predictors of risk for type 2 diabetes mellitus and impaired glucose tolerance in polycystic ovary syndrome: a prospective, controlled study in 254 affected women. J Clin Endocrinol Metab. 1999;84(1):165-9.

28. Tomlinson JW, et al. Impaired glucose tolerance and insulin resistance are associated with increased adipose 11 beta-hydroxysteroid dehydrogenase type 1 expression and elevated hepatic 5alpha-reductase activity. Diabetes. 2008:57(10):2652-60.

Submit your next manuscript to BioMed Central and we will help you at every step:

- We accept pre-submission inquiries

- Our selector tool helps you to find the most relevant journal

- We provide round the clock customer support

- Convenient online submission

- Thorough peer review

- Inclusion in PubMed and all major indexing services

- Maximum visibility for your research

Submit your manuscript at www.biomedcentral.com/submit
Biomed Central 